\title{
CONDITIONING EFFECTS OF BACKWARD TREADMILL TRAINING IN CHILDREN WITH SPASTIC DIPLEGIC CEREBRAL PALSY
}

\section{Doaa Ahmed Mahmoud Sanad.}

Lecturer, Department of Pediatric Physical Therapy, Faculty of Physical Therapy, Cairo University, Egypt.

\section{ABSTRACT}

Background: A new way to increase the challenge of walking is to walk backwards. Backward treadmill walking may provide advantages by promoting improvement in balance, walking spatiotemporal parameters and quality that may reflect in improving walking speed. Information about the energy expended by the patient during ambulation could provide objective data to help guide the decision-making process.

Objective: To evaluate the effects of a backward treadmill training program in improving walking endurance in children with cerebral palsy.

Materials and Methods: Twelve children diagnosed with spastic diplegic cerebral palsy from both gender were included in this study. They were divided into 2 groups, group $A$ (control) received the regular therapeutic exercise program according to neurodevelopmental approach for such cases and group B (study) received the regular therapeutic exercise program for such cases along with 20 min backward treadmill walking using Biodex gait trainer equipment. Heart rate was monitored continuously each session for both groups. walking speed was assessed using The Biodex Gait Trainer 2TM and energy expenditure index was assessed by measuring heart rate using pulsometer (Japan model Tunturi TPN-400) for both groups before and after three months of the treatment program.

Results: There was statistically significant improvement in walking speed in the study group $(P<0.05)$ with significant difference when comparing post treatment results between groups $(p<0.05)$, but The change in mean EEI scores was not significant when comparing post treatment results between groups while there were a significant improvement in mean EEI scores within groups.

Conclusion: This study demonstrated an effective protocol using backward treadmill training to increase walking speed and endurance in children with CP. Although no significant change in EEI scores was noted and the sample size was small. This gait training method provides a safe and controlled environment for children to perform continuous walking at a cardiovascular training intensity to improve their gait and fitness.

KEY WORDS: Backward, Treadmill Training, Energy Expenditure Index, Diplegia, walking speed, Cerebral Palsy.

Address for correspondence: Dr. Doaa Ahmed Mahmoud Sanad, Ph.D, Lecturer, Department of Pediatric Physical Therapy, Faculty of Physical Therapy, Cairo University, Egypt.

Telephone:+201007206392 E-Mail: dr.doaa.sanad@gmail.com \doaa.mahmoud@pt.cu.edu.eg

\section{Access this Article online}

\section{Quick Response code}

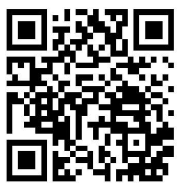

DOI: $10.16965 /$ ijpr.2017.194
International Journal of Physiotherapy and Research

ISSN 2321- 1822

www.ijmhr.org/ijpr.html

$\begin{array}{ll}\text { Received: 29-06-2017 } & \text { Accepted: 02-08-2017 } \\ \text { Peer Review: 30-06-2017 } & \text { Published (O): 11-09-2017 } \\ \text { Revised: None } & \text { Published (P): 11-10-2017 }\end{array}$

\section{INTRODUCTION}

Spastic diplegia is a type of cerebral palsy that generally affects the lower extremities greater than the upper extremities. Gait is altered in individuals with spastic diplegia CP due to the increase in muscle tone causing legs to adduct, internally rotate, and causes a "scissor" gait pattern when ambulating (American Physical Therapy Association, 2001), ("Facts about Cerebral Palsy," 2015) [1].

It was reported that repetitive gait training was more effective in improving gait speed, stride 
length, and joint kinematics than traditional physical therapy in children with cerebral palsy [2]. Traditional treadmill gait training, which is one type of commonly used repetitive gait training, improves motor learning and lower limb muscle strength, activates locomotor control systems, and enables children to experience the habits and task-specific gait behaviors that affect functional ability [3,4].

A relatively new way to increase the challenge of walking is to walk backwards. At a given speed, when compared with forward walking, backward walking elicits more electromyographic activity which in turn results in higher physiological cost and greater perceived exertion $[5,6]$. Hip extension is more active in backward walking due to the concentric contraction of knee flexors during early swing phase $[7,8]$ which may be beneficial at improving lower limb coordination. Also, backward walking is more difficult and demanding than forward walking due to its postural instability and may therefore provide a training challenge to balance [9].

One study showed that when healthy schoolaged children engaged in backward gait, their sense of balance improved [10]; moreover, backward gait at the same speed consumed more oxygen, increased metabolic rate, and enhanced cardiovascular function compared to forward gait [6]. As backward walking has a good effect on acting to improve proprioception and balance. This has a role in increasing speed of walking as when the patient becomes balanced so this will also give him greater support to move faster and will act to condition hip, knee and ankle stabilizers. This increase at the speed of walking is related also to the increase at lower limb activity that occurs with backward walking as when the activity of lower limb muscles is improved so the patient can move at faster speed [6].

The energy expenditure index (EEI) represents a ratio of resting and walking heart rate to walking velocity and results in units of beats per meter [11] and has been used as an indicator of energy expenditure for children with cerebral palsy in past research $[12,13]$.

Heart rate is an easily measured parameter. Although heart rate may be affected by factors other than oxygen uptake, it has been shown to be an accurate and convenient index of energy expenditure for children during a steady state of submaximal work. Heart rate has been suggested to estimate energy expenditure in normal and disabled children [14].

Walking energy consumption is a valid evaluation of walking performance in individuals. Gait disabilities, such as CP and stroke, increase the energy expenditure of walking and the intensity of physical effort with altered walking speeds [15]. To our knowledge, no published research was specifically designed to assess cardiorespiratory fitness performance in children with CP. To address this gap in the research literature, the current study was designed to evaluate the effect of backward treadmill walking exercise as a mean of improving the energy expenditure of walking and gait efficiency in children with spastic diplegic CP. We also determined improvement in one parameter such as gait speed during backward treadmill walking in response to pre treatment measure.

\section{MATERIALS AND METHODS}

Subjects: Twelve subjects diagnosed with spastic diplegic cerebral palsy from both gender were included in this study, they were selected from out- patient clinic, Faculty of Physical Therapy, Cairo University. The criteria for selection of the subjects were as follows: Their ages ranged from five to nine years; Gross Motor Function Classification System (GMFCS) levels I-III; Modified Ashworth Scale (MAS) levels 1-2; able to walk $10 \mathrm{~m}$ backward using an assistive gait device; Their heights were 1 meter and more to be able to see the screen and they had abnormal gait kinematics which can be collected from assessment of gait kinematics by Biodex Gait Trainer II ${ }^{\mathrm{TM}}$ and able to follow verbal instructions. The criteria for exclusion are as follows: inability to walk independently; neurological or orthopedic surgery related to cerebral palsy within the prior six months; uncontrollable seizures, or visual, auditory, or perception difficulty. They were divided into 2 groups, group A (four boys and two girls) (control) received the regular therapeutic exercise program according to neurodevelop-mental approach for such cases) and group B (four boys and two girls) (study) received the regular therapeutic exercise program for such cases along 
with 20 min backward treadmill walking using Biodex gait trainer equipment. Heart rate was monitored continuously each session for both groups.

Materials: Clinical assessments included one measure of endurance (Energy Expenditure Index) and one functional gait measure (Walking Velocity) under the direction of a physical therapist.

For the EEI measurement for diplegic children: The (EEI) in beats/meter is calculated as the ambulation heart rate (beats/min) minus the resting heart rate (beats/min) divided by the ambulation velocity (meters/min). Higher numbers indicate greater energy expenditure, while lower numbers reflect more energy efficiency [16]. The heart rate measured by using pulsometer (Japan model Tunturi TPN-400)

To assess walking speed for the children participated in this study: The Biodex Gait Trainer 2TM is equipment used to evaluate and provide training of walking in patients with gait disorders. (Biodex Medical INC., Shirley, New York, USA).

Methods: After a clear explanation of the study protocol and written consents were obtained from all participants and their parents or care givers, the ethics committee, Faculty of Physical Therapy, Cairo University approved this study.

For treatment, the children in the control group (A) received the traditional physical therapy program based on NDT only for one hour three times /week for 12 weeks while Children of study group (B) received the traditional physical therapy program based on NDT for such cases in addition to walking backward on a treadmill using The Biodex Gait Trainer with speed of 0.01 $\mathrm{m} / \mathrm{sec}$. and 0 degree inclination for $5 \mathrm{~min}$. firstly increased gradually to reach $2 \mathrm{~m} / \mathrm{sec}$. for total time of session 20 min [17], this program was conducted three times / week for three successive month

For evaluation all children were evaluated prior and after three-months of training. For evaluation of EEI, Firstly, The child rested seated in silence for about ten minutes and the mean heart rate was taken at $2^{\text {nd }}$ minute, fifth min. and eighth min. as the resting heart rate by using pulsometer. The walking heart rate was measu- red on the treadmill using pulsometer and the mean heart rate was taken at the last three minutes of work during the last session of second and third month of training to assess endurance. Treadmill walking was adjusted at the predetermined individual, self-selected speed, which was held constant throughout the test. For evaluation of walking speed, each child was permitted to try the gait trainer set up before recording his gait parameters.

Statistical Analysis: For data analysis, all statistical measures were performed through the Statistical Package for Social Studies (SPSS) version 17 for windows, (SPSS, Inc., Chicago, IL). Paired and unpaired $T$ test were performed to detect level of significance within and between groups respectively.

\section{RESULTS}

This randomized controlled study was conducted to detect the conditioning effect of backward gait training in addition to the physical therapy program in children with diplegic cerebral palsy.

There were two independent variables, the first one was the tested groups which had two groups, group A (received the regular therapeutic exercise program for such cases based on neurodevelopmental therapy) and group B (received the regular therapeutic exercise program for such cases besides backward gait training).

The second one was the (training periods); within subject factor which had two levels (pre, post). In addition, this test involved two dependent variables (energy expenditure index (EEI) in beats/ minute and walking speed in meter/ sec. ).

Data were obtained from patients of the two groups (control and study), statistically analyzed and compared with measurable variable [energy expenditure index (EEI) in beats/ minute] obtained before and after treatment; using paired and unpaired T- test to detect level of significance within and between groups respectively.

\section{Energy Expenditure Index (EEI):}

Within Groups: As presented in table (1) and illustrated in figure (1), within group's comparison the mean $\pm S D$ values of energy expenditure index (EEI) in the "pre" and "post" tests were $1.91 \pm 0.92$ and $1.86 \pm 0.89$ (Beats/Min) 
respectively in the group (A). which revealed no significant difference ( $P$-value $>0.05$ ).

It's also presented in table (1) and illustrated in figure (1), within group's comparison the mean \pm SD values of energy expenditure index in the "pre" and "post" tests were 2.08 \pm 0.75 and $1.32 \pm 0.55$ (Beats/Min) respectively in the group (B), which indicated a significant difference $(P<0.05)$.

Between Groups: Date presented in Table 1 and figure 1 showed that, the pre treatment mean values + SD of energy expenditure index (EEI) for group $A$ and $B$ were $1.91 \pm 0.92$ and $2.08 \pm 0.75$ (Beats/Min) respectively. The differences between pre treatment mean scores + SD of the energy expenditure index (EEI) indicated no significant differences $(P>0.05)$.

Also, date presented in table (1) and figure (1) showed that, the post treatment mean values + SD of EEI for group A and B were $1.86 \pm 0.89$ and $1.32 \pm 0.55$ (beats $/ \mathrm{min}$ ) respectively. The differences between post treatment mean scores + $\mathrm{SD}$ of the energy expenditure index (EEI) indicated no significant differences $(P>0.05)$.

Table 1: Pre and post treatment mean values of energy expenditure index (beats/min) for both groups (A and B).

\begin{tabular}{|c|c|c|c|c|c|c|}
\hline $\begin{array}{c}\text { EEI } \\
\text { (Beats/Min) }\end{array}$ & Means \pm SD & Means \pm SD & MD & $\begin{array}{c}\text { \% of } \\
\text { change }\end{array}$ & T value & P-value \\
\cline { 2 - 6 } & Pre test & Post test & & & \\
\hline Group A & $1.91 \pm 0.92$ & $1.86 \pm 0.89$ & 0.05 & $2.62 \downarrow$ & 0.34 & 0.38 \\
\hline Group B & $2.08 \pm 0.75$ & $1.32 \pm 0.55$ & 0.76 & $36.54 \downarrow$ & 4.91 & $0.002^{*}$ \\
\hline P value & 0.73 & 0.23 & & & & \\
\hline
\end{tabular}

*Significant level is set at alpha level $<0.05$.

Fig. 1: Pre and post treatment mean values of energy expenditure index (beats/min) for both groups (A \& B ).

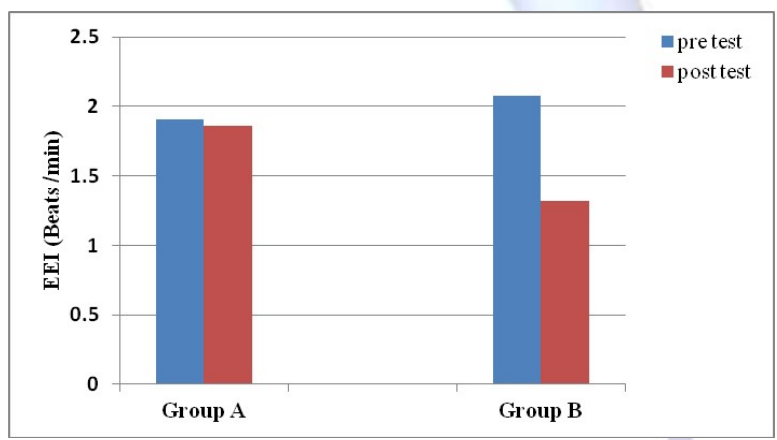

\section{Walking speed:}

Within Groups: As presented in table (2) and illustrated in figure (2), within group's comparison the mean \pm SD values of walking speed in the "pre" and "post" tests were $0.29 \pm 0.15$ and
$0.31 \pm 0.14$ (Meter/sec) respectively in the group (A). which revealed no significant difference (P-value $>0.05)$. It's also presented in table (2) and illustrated in figure (2), within group comparison the mean \pm SD values of walking speed in the "pre" and "post" tests were $0.34 \pm 0.16$ and $0.52 \pm 0.16$ (Meter/sec) respectively in the group (B). which indicated a significant difference $(P<0.05)$.

Between Groups: Date presented in table (2) and figure (2) showed that, the pre treatment mean values $+S D$ of walking speed for group $A$ and $B$ were $0.29 \pm 0.15$ and $0.34 \pm 0.16$ (Meter/Sec) respectively. The differences between pre treatment mean scores + SD of the walking speed indicated no significant differences $(P>0.05)$.

Also, date presented in table (2) and figure (2) showed that, the post treatment mean values + SD of walking speed for group $A$ and $B$ were $0.31 \pm 0.14$ and $0.52 \pm 0.16$ (Meter/Sec) respectively. The differences between post treatment mean scores + SD of the walking speed revealed a significant differences $(P<0.05)$.

Table 2: Pre and post treatment mean values of walking speed in both groups ( $A$ and $B$ )

\begin{tabular}{|c|c|c|c|c|c|}
\hline $\begin{array}{c}\text { Walking } \\
\text { speed }\end{array}$ & Means \pm SD & Means $\pm S D$ & \multirow{2}{*}{ MD } & $\begin{array}{c}\% \text { of } \\
\text { change }\end{array}$ & P-value \\
\cline { 2 - 3 } & Pre test & Post test & & \\
\hline Group A & $0.29 \pm 0.15$ & $0.31 \pm 0.14$ & 0.018 & 6.24 & 0.59 \\
\hline Group B & $0.34 \pm 0.16$ & $0.52 \pm 0.16$ & 0.182 & $53.5 \uparrow$ & $0.000^{*}$ \\
\hline P-value & 1 & $0.036^{*}$ & & & \\
\hline
\end{tabular}

*Significant level is set at alpha level $<0.05$

Fig. 2: Pre and post treatment mean values of walking speed (Meter/sec) in both groups ( $A$ and $B$ ).

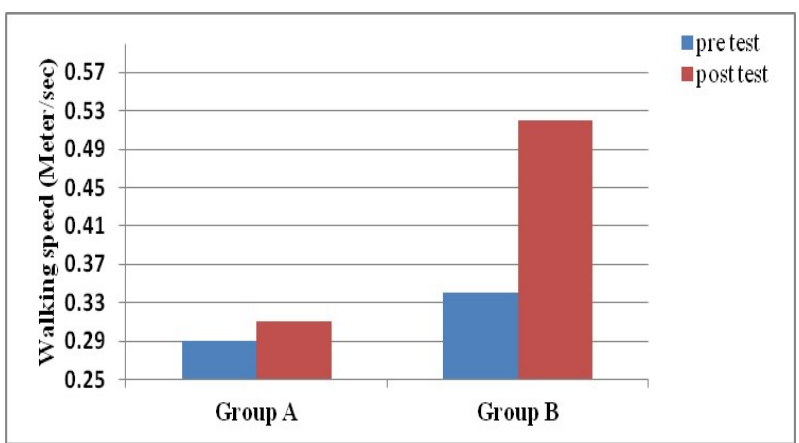

\section{DISCUSSION}

The aims of this study were to evaluate the effects of a backward treadmill training program in improving energy cost during walking in children with spastic diplegic cerebral palsy; walking capacity has been shown to predict physical activity level and community participation. Because backward walking is a challeng 
ing activity, the results of this trial may produce important advances in walking rehabilitation and increased independent community walking in children with spastic diplegic cerebral palsy.

A major movement related limitation for children with spastic cerebral palsy is the compromised gait pattern, which may explain their excessive energy cost of locomotion. As suggested by previous studies that individuals with $\mathrm{CP}$ are less active and more sedentary than their able-bodied peers [18]. Children and adolescents with CP tend to participate in less structured and lower intensity physical activities. A vicious cycle of exposure to lower physical activities in persons with chronic illness or disability leads to further deconditioning and physical deterioration [19]. Children with $\mathrm{CP}$ at the age of 7 have already lowered $\mathrm{VO}_{2}$ peak values compared to typical developing peers. Therefore, early intervention for an active lifestyle might be beneficial for promoting physical fitness in CP patients [20].

Observation of the pre-treatment mean values of measured variables of this study revealed abnormal gait characteristics associated with CP that could be associated with elevated energy cost: toe strike, or toe-heel strike due to posterior calf spasticity, shortened step length on the affected side, abnormal arm swing limiting ability to carry out reciprocating gait, and the excessive absorption of eccentric work by the knee extensors almost as soon as it was generated by ankle plantar flexors with cocontraction of agonist and antagonist muscle, all of that leading to decreased walking speed with high energy expenditure, this result confirmed by the result of Dahlback and Rorlin [21] who stated that Gait abnormalities in children with cerebral palsy have been shown to increase submaximal walking energy expenditure almost three-fold compared with healthy children . One consequence of this increased energy cost is that children with CP complain of fatigue at low walking intensities.

The explanation for this phenomenon remains incomplete. One factor that has been implicate, but not documented, is the amount of simultaneous contraction of agonist and antagonist muscle. Such"cocontraction"can be both beneficial and detrimental. It is a way of achieving joint stability, particularly at the ankle and knee joints and specifically during locomotion. However, in instances when the antagonist muscle is activated excessively, movement is achieved at a high metabolic cost, reflecting the effort of both muscles [22].

The significant improvement obtained in the post-treatment mean values of the variable related to walking speed especially for the study group may be attributed to the effect of backward gait treadmill training in ambulatory $\mathrm{CP}$ patients who have higher energy expenditure and lower gait efficiency, this finding come in agreement with Schindl et al. [23] who reported that effects of treadmill walking exercise as a treatment method to improve the physical fitness in children with $\mathrm{CP}$ who were mostly nonambulatory; their data demonstrate relevant improvements in motor abilities.

Also this finding confirmed by the result of Duffy et al. [24] who demonstrated that the $O 2$ cost was significantly higher in children with spina bifida and CP compared to healthy children. In this study, ambulatory CP patients in the experimental group increased their walking distance and gait velocity, leading to a decrease in the $\mathrm{O} 2$ cost during overground walking after treadmill walking exercise. It suggested that treadmill walking exercise characteristically improved the gait efficiency during overground walking.

Significant Improvement of walking speed in study group children is duo to effect of backward gait training in improving proprioception and balance. This has a role in increasing speed of walking as when the patient becomes balanced so this will also give him greater support to move faster. This is supported by Hooper et al. [6] who reported that backward walking improves the patient's balance and proprioception and will act to condition hip, knee and ankle stabilizers. This increase at the speed of walking is related also to the increase at lower limb activity that occurs with backward walking as when the activity of lower limb muscles is improved so the pa-tient can move at faster speed. Recent evidence indicates that when the direction of walking is the same for the 2 conditions, similar neural networks may be involved; however, when on the treadmill, subjects do not move through space as they do during over 
ground walking. These contextual differences arise from the lack of visual and somatosensory information during treadmill walking. It is possible that these identifiable differences in task context are sufficient to transfer from the treadmill walking exercise to a more natural overground walking environment with more efficient gait [25].

The finding of this study related to (EEI) variable indicated no significant difference between post treatment mean scores + SD of the energy expenditure index (EEI) between both groups, there were decreasing in mean value that revealed improvement in energy cost during gait but not significantly as a result of the effect of backward gait training, otherwise the mean \pm $S D$ values of energy expenditure index in the "pre" and "post" tests in the study group (B), which indicated a significant difference $(P<$ $0.05)$, it may be attributed to the difference in age and physical fitness between subjects, this finding come in agreement with the finding of Green and Foster [26] who demonstrated that the level of reduction in oxygen cost of treadmill exercise depends upon the degree by which the subjects grasp the handrails. It has been clearly demonstrated in the comparison of cardiorespiratory measures among different age groups of children that growth differences can give rise to differences in running and walking energy expenditure. Hence, to demonstrate cardiorespiratory differences resulting from a disability (CP) rather than the confounding effects of growth, the subjects need to be similar in age, height, and mass. In the Rose et al. [27] study the groups differed in mass and height, and there was no indication of any between-groups statistical comparison. In addition, the fitness levels of the children with CP were not documented in any of the studies by Rose et al. [27].

Non significant improvement in energy cost during gait between children with $\mathrm{CP}$ in study and control group may be come from the higher energy expenditures associated with involuntary local muscle activity, this confirmed by the result of Berger [28] who stated that the source of the pathological pattern of muscle activity seen in the children with $\mathrm{CP}$ is open to speculation. It could be a consequence of an impaired maturation of the locomotor pattern and neu- ronal adaptation to altered peripheral muscle function in early infancy before walking is learned. Alternatively, as a result of the insult to the brain stem and cerebellum at birth, normal inhibition patterns could be affected. The absence of normal inhibition patterns gives rise to unsuccessful repression of undesired contractions generating excessive cocontraction levels, abnormal gait, and a marked increase in energy consumption. While the higher oxygen cost of walking can be explained by cocontraction in the children with $\mathrm{CP}$, the same cannot be said for the control subjects. As reported by Gatev and Sutherland et al. $[22,29]$ there appears to be a certain amount of cocontraction that is beneficial for joint stability and that is associated with a certain $\mathrm{O} 2$ cost. Beyond this level, any additional cocontraction may induce an excessively high oxygen cost of walking.

Finally, backward treadmill walking exercise improved gait efficiency in children with spastic diplegic cerebral palsy. It has been associated with some decrease in energy expenditure during overground walking.

This study had some limitations that need to be acknowledged, such as the small sample size.

\section{CONCLUSION}

According to the results of this study, it can be concluded that backward treadmill training was found to be an effective intervention to improve cardiovascular endurance and walking speed in a cohort of children with spastic diplegic cerebral palsy.

\section{Conflicts of interest: None}

\section{REFERENCES}

[1]. Facts about cerebral palsy (2015, January 12). Retrieved February 28, 2015, from http://www.cdc.gov/ ncbddd/cp/facts.html.

[2]. Smania N, Bonetti P, Gandolfi M, et al. : Improved gait after repetitive locomotor training in children with cerebral palsy. Am J Phys Med Rehabil, 2011;90:137-149. [PubMed].

[3]. Richards CL, Malouin F, Dumas F, et al. : Early and intensive treadmill locomotor training for young children with cerebral palsy: a feasibility study. Pediatr Phys Ther, 1997;9:158-165.

[4]. Takao T, Tanaka N, lizuka N, et al. Improvement of gait ability with a short-term intensive gait rehabilitation program using body weight support treadmill training in community dwelling chronic post stroke survivors. J Phys Ther Sci, 2015;27:159-163. [PMC free article] [PubMed]. 
[5]. Grasso R, Bianchi L, Lacquaniti F. Motor patterns for human gait: backward versus forward locomotion. J Neurophysiol 1998;80:1868-85.

[6]. Hooper TL, Dunn DM, Props JE, Bruce BA, Sawyer SF, Daniel JA. The effects of graded forward and backward walking on heart rate and oxygen consumption. J Orthop Sports Phys Ther 2004;34:65-71.

[7]. Winter DA, Pluck N, Yang JF. Backward walking: a simple reversal of forward walking? J Mot Behav 1989;21:291-305.

[8]. Thorstensson A. How is the normal locomotor program modified to produce backward walking? Exp Brain Res 1986;61:664-8.

[9]. Katsavelis D,Mukherjee M, Decker L, Stergiou N. Variability of lower extremity joint kinematics during backward walking in a virtual environment. Nonlinear Dynamics Psychol Life Sci 2010;14:16578.

[10]. Flynn TW, Connery SM, Smutok MA, et al. Comparison of cardiopulmonary responses to forward and backward walking and running. Med Sci Sports Exerc, 1994;26:89-94. [PubMed].

[11]. Rose, J., Medeiros, J.M., \& Parker, R. Energy cost index as an estimate of energy expenditure of cerebral-palsied children during assisted ambulation. Developmental Medicine and Child Neurology, 1985;27:485-490.

[12]. Eagleton, M., lams, A., McDowell, J., Morrison, R., \& Evans, C.L. The effects of strength training on gait in adolescents with cerebral palsy. Pediatric Physical Therapy, 2004;16:22-30.

[13]. Schlough, K., Nawoczenski, D., Case, L.E., Nolan, K., $\&$ Wigglesworth, J.K. The effects of aerobic exercise on endurance, strength, function and self- perception in adolescents with spastic cerebral palsy: a report of three case studies. Pediatric Physical Therapy, 2005;17:234-250.

[14].Cooper DM, Weiler-Ravell D, Whipp BJ and Wasserman K. Growth related changes in Oxygen uptake and heart rate during progressive exercises in children. Pediat Res 1984;18:845-51.

[15]. Dal U, Erdogan T, Resitoglu B, Beydagi H. Determination of preferred walking speed on treadmill may lead to high oxygen cost on treadmill walking. Gait Posture. 2010;31:366-369. [PubMed].

[16]. Rose, J., Gamble, J.G. and Lee, J. The energy expenditure index: a method to quantitate and compare walking energy expenditure for children and adolescents. J Pediatr Orth., 1991;11:571-578.

[17]. Hesse SA., Bertlt CM., Schaffrin A. Restoration of gait in non ambulatory hemiparetic patients by treadmill training with partial body-weight support. Arch. Phys. Med. Rehabil. , 2009;75(10):1087-1093.
[18]. Maher CA, Williams MT, Olds T, Lane AE. Physical and sedentary activity in adolescents with cerebral palsy. Dev Med Child Neurol., 2007;49:450-457. [PubMed].

[19]. Durstine JL, Painter P, Franklin BA, Morgan D, Pitetti $\mathrm{KH}$, Roberts SO. Physical activity for the chronically ill and disabled. Sports Med. ,2000;30:207219. [PubMed].

[20]. Verschuren O, Takken T. Aerobic capacity in children and adolescents with cerebral palsy. Res Dev Disabil. ,2010;31:1352-1357. [PubMed].

[21]. Dahlback, G. O. and R. Rorlin. The effect of corrective surgery on energy expenditure during ambulation in children with cerebral palsy. Eur. J. Appl. Physiol., 1985;54:67-70. Full Text Internet Resources Bibliographic Links [Context Link].

[22]. Sutherland, D. H., R. Olshen, L. Cooper, and S. Woo. The development of mature gait. J. Bone Joint Surg., 1980;62A:336-353. Internet Resources Bibliographic Links [Context Link].

[23]. SchindI MR, Forstner C, Kern H, Hesse S. Treadmill training with partial body weight support in nonambulatory patients with cerebral palsy. Arch Phys Med Rehabil., 2000;81:301-306. [PubMed].

[24]. Duffy CM, Hill AE, Cosgrove AP, Corry IS, Graham HK. Energy consumption in children with spina bifida and cerebral palsy: a comparative study. Dev Med Child Neurol. ,1996;38:238-243. [PubMed].

[25]. Reisman DS, Wityk R, Silver K, Bastian AJ. Split-belt treadmill adaptation transfers to overground walking in persons poststroke. Neurorehabil Neural Repair , 2009;23:735-744. [PMC free article] [PubMed].

[26]. Green, M. A. and C. Foster. Effect of magnitude of handrail support on prediction of oxygen uptake during treadmill testing (Abstract). Med. Sci. Sports Exerc., 1991;23:S166. Internet Resources Bibliographic Links [Context Link].

[27]. Rose, J., W. L. Haskell, and J. G. Gamble. A comparison of oxygen pulse and respiratory exchange ratio in cerebral palsied and nondisabled children. Arch. Phys. Med. Rehabil.,1993;74:702-705. Full Text Internet Resources Bibliographic Links [Context Link].

[28]. Berger, W., J. Quintern, and V. Dietz. Pathophysiology of gait in children with cerebral palsy. Electroencephalogr. Clin. Neurophysiol. , 1982;53:538-548. Full Text Internet Resources Bibliographic Links [Context Link].

[29]. Gatev, V. Role of inhibition in the development of motor coordination in early childhood. Develop. Med. Child Neurol. 1972;14:336-341. Internet Resources Bibliographic Links [Context Link].

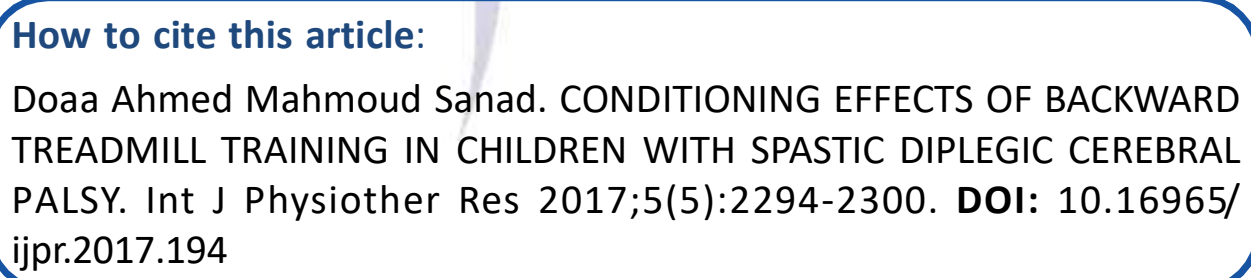

